\title{
Surface-Enhanced Raman Analysis of Underlaying Colorants On Re-Dyed Hair
}

Isaac Esparza ${ }^{1}$, Rui Wang ${ }^{1}$ and Dmitry Kurouski ${ }^{1,2 *}$

1. Department of Biochemistry and Biophysics, Texas A\&M University, College Station, Texas 77843, United States

2. The Institute for Quantum Science and Engineering, Texas A\&M University, College Station, Texas, 77843, USA.

\section{Supporting Information}

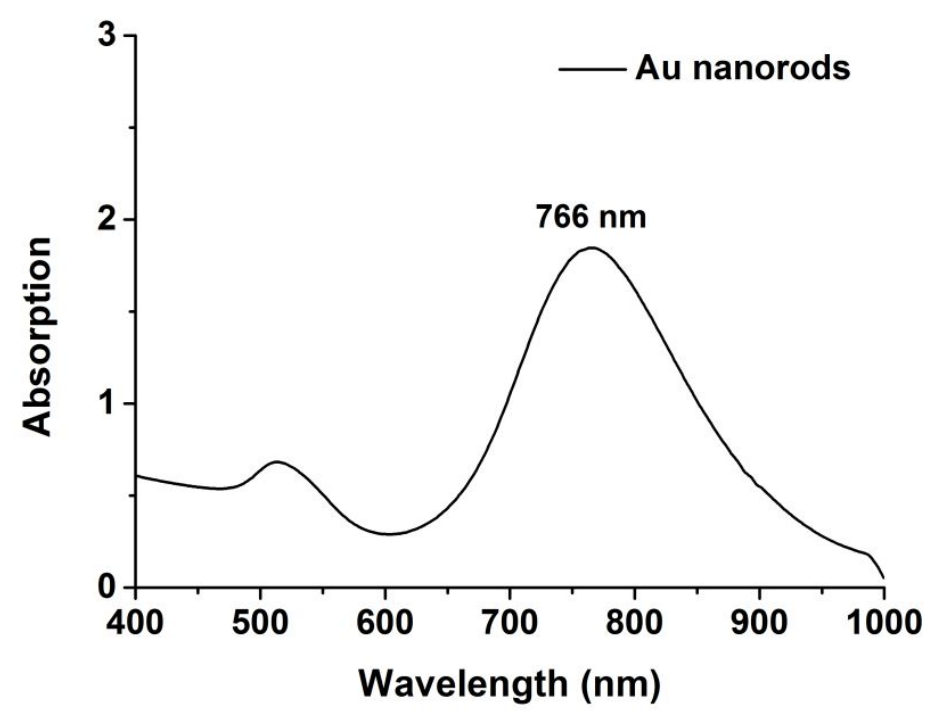

Figure S1. Absorption spectrum of purified Au nanorods 

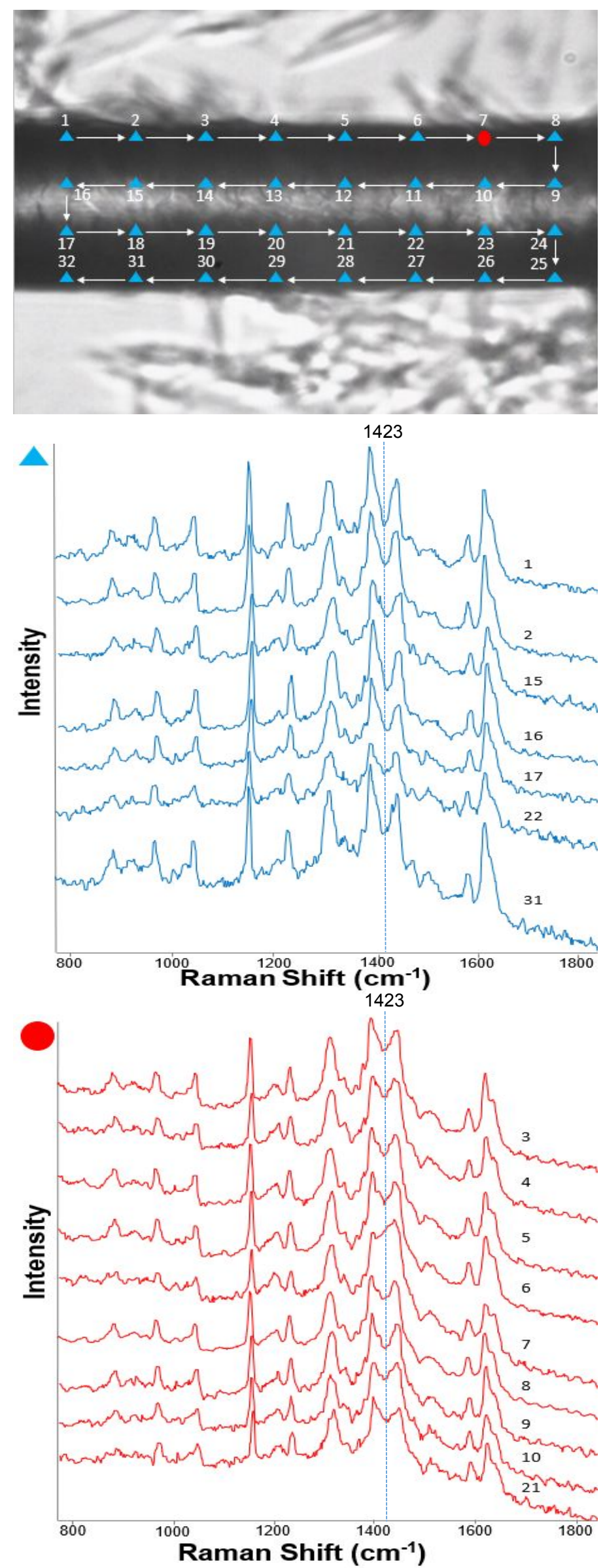

Figure S2. Microphotograph of a single hair dyed with $\mathrm{BLK}^{\mathrm{P}} \rightarrow \mathrm{BLU}^{\mathrm{SP}}$ with 
schematically shown spectral collection pattern. In total, 32 spectra (4 lines with 8 spectra per line) were acquired, which represents $96 \times 160 \mu \mathrm{m}$ surface area. Some of the collected Raman spectra exhibit vibrational fingerprint that corresponds to BLU ${ }^{S P}$ (shown in blue), whereas other spectra had vibrational fingerprints of both $\mathrm{BLK}^{\mathrm{P}}$ and BLU $^{S P}$ (shown in red). 